\title{
Primary intraosseous lymphoma at right femur
}

\author{
Upadhyaya $\mathrm{P}^{1}$, Karki $\mathrm{S}^{1}$, Paudyal $\mathrm{P}^{1}$,Shrestha $\mathrm{G}^{1}$, Shrestha $\mathrm{A}^{1}$, Bastakoti $\mathrm{S}^{1}$ \\ ${ }^{1}$ Department of Pathology, B.P. Koirala Institute of Health Sciences, Dharan, Nepal.
}

\author{
Keywords: \\ Intraosseous; \\ Lymphoma; \\ CD20; \\ BCL2; \\ Diffuse large B cell.
}

\begin{abstract}
Lymphomas, the malignant neoplasms of the reticuloendothelial and lymphatic system, are classified based on characterisitic morphological pattern, immunophenotypic pattern and sometimes distinctive chromosomal aberrations. Most of the intraosseous lymphomas should be metastatic, and primary intraosseous lymphoma is a rare type of lymphoma, which accounts for only about $5 \%$ extranodal lymphoma. It accounts for approximately $3 \%$ of all primary bone malignancies.

We report a case of 54 years lady presented with pain over right thigh since 3 to 4 months which suddenly increased. The clinico-radiological differential diagnosis was fibrous dysplasia, plasmacytoma or metastasis.

The histological findings and immunohistochemistry being positive for CD20 and BCL2 negative are those of Lymphoma- Diffuse large B cell type. Radiotherapy and/or chemotherapy is the preferred modality of management.
\end{abstract}

\section{INTRODUCTION}

Lymphomas, the malignant neoplasms of the reticuloendothelial and lymphatic system, are classified based on characteristic morphological pattern, immunophenotypic pattern and sometimes distinctive chromosomal aberrations. They have been traditionally divided into Hodgkin Disease and Non Hodgkin Lymphoma because of their differences in histology, pattern of behavior, immunohistochemical findings and cytogenetics. ${ }^{1}$ Primary Bone lymphoma (PBL) was first described as a distinct clinicopathological entity in 1939 by Parker and Jackson. ${ }^{2}$ Intraosseous Lymphoma can be considered as metastatic lymphoma, or as primary

\section{Correspondence:}

Dr. Paricha Upadhyaya, MBBS, $M D$

Additional Professor, Department of Pathology

B.P. Koirala Institute of Health Sciences, Dharan, Nepal

Email:paricha7@yahoo.com bone lymphoma which developed in the bone without involvement of any other site in the body. ${ }^{3}$

Most of the intraosseous lymphomas should be metastatic, and primary intraosseous lymphoma is a rare type of lymphoma, which accounts for only about 5\% extranodal lymphoma. It accounts for approximately $3 \%$ of all primary bone malignancies. In contrast to the classical manifestations of patients with non-bone lymphoma, symptoms, including fever, body weight losses, and night sweating, are rarely noted among patients with primary bone lymphoma. The most common presentation of the patients with intraosseous lymphoma is bone pain and, less frequently, with a soft-tissue swelling or a palpable mass. Primary Bone lymphoma can involve any part of the skeleton, but a trend exists in favour of the bones with persistent bone marrow. ${ }^{5}$ 


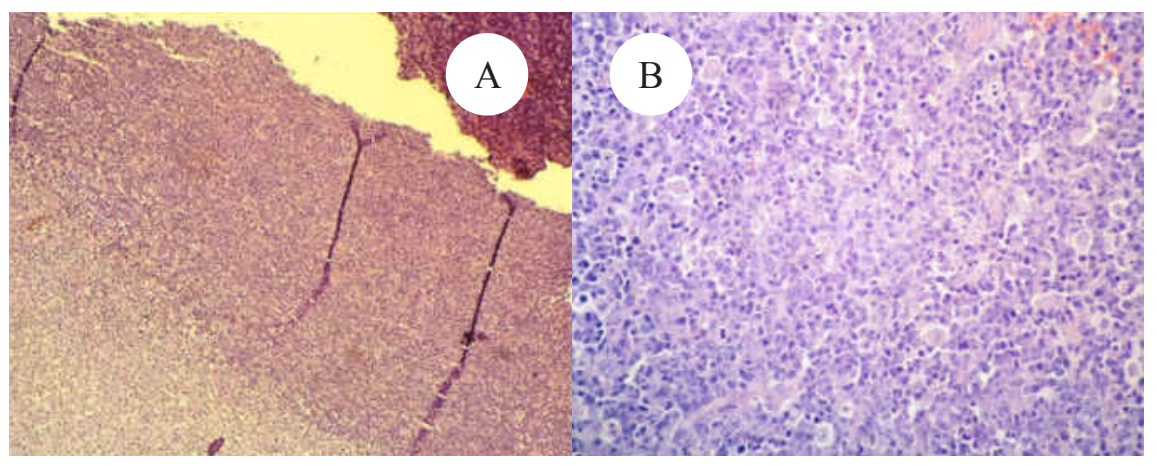

Figure $1(\boldsymbol{A}, \boldsymbol{B})$ : Atypical intermediate to large sized lymphoid cells; rounded to cleaved nuclei; patchy areas of necrosis. A (HE stain, X40), B. HE stain, X400)

Primary Bone lymphoma occurs commonly between 20 and 50 years of age and it shows a male preponderance with a male to female ratio of $3: 2 .{ }^{4}$

Diffuse large-B-cell lymphoma (DLBCL) accounts for the majority of cases of PBL. ${ }^{5}$ Appropriate treatment depends on the histologic type and stage. Traditionally, irradiation has been used alone or in combination with chemotherapy. ${ }^{6}$

\section{CASE REPORT}

A 54-years-old lady presented with pain over right thigh since 3 to 4 months which suddenly increased without inciting factors. The clinico- radiological differential diagnosis was fibrous dysplasia, Plasmacytoma or Metastasis and there was no organomegaly and lymphadenopathy. In Department of Surgical Pathology, specimen was submitted as multiple bits of bone and content of medullary canal, measuring altogether $6 \times 5.5 \times 1.2 \mathrm{~cm}$. Histological examination revealed multiple tissue bits composed predominantly of areas of necrosis and fragments of dead bony specules. The tumors was composed of monotonous population of atypical lymphoid cells of intermediate to large size and have rounded to cleaved nuclei with prominent nucleoli and scant amount of cytoplasm and were arranged in diffuse sheet punctuated by scattered tingible body macrophages. These atypical cells were seen surrounding central area of coagulative necrosis along with fibrin (fig. 1A\&B). Immunohistochemisty was positive for CD20 (fig.2) and negative for Bcl6. The histopathothological features supported by immunohistochemistry was those of Lymphoma- Diffuse Large B-cell type.

\section{DISCUSSION}

Primary lymphoma of bone is rare, and most lymphomas that involve bone are metastatic. The clinical presentation includes local pain, swelling and sometimes even a pathological fracture. The diagnosis is established by biopsy. ${ }^{4}$ The histological features of primary nonHodgkin's lymphoma of the bone are identical to those of

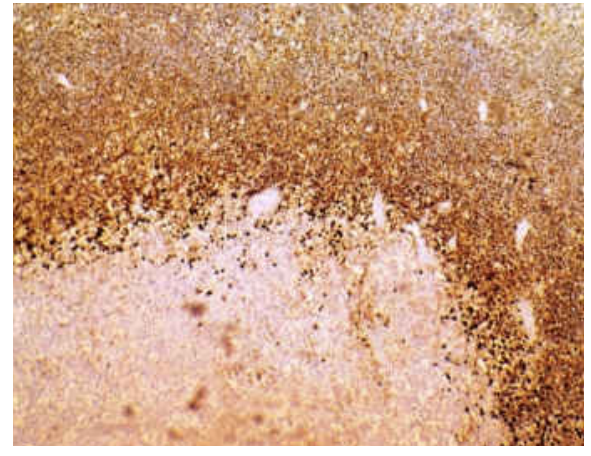

Figure 2: CD 20 positive with areas of necrosis not taking CD20 (IHC, X40).

the conventional, non-osseous forms of non-Hodgkin's lymphoma. The hallmark attribute of the lesion is a replacement of the normal marrow constituents by neoplastic lymphoid tissue; the most common subtype is diffuse large B-cell lymphoma.

In order to be diagnosed as PLB the following criteria (known as Coley's criteria) should be met. ${ }^{5}$

- A primary focus in a single bone

- Positive histological diagnosis

- No evidence of distal soft tissue or distal nodal involvement

In the recent study that reviews a total of 60 cases of bone lymphoma during the period from 1998 to 2008, the most common site of involvement was femur (28.3\%), followed by hip (16.6\%) and humerus $(10 \%) .^{7}$ In addition, iliac bone and spine were ever reported to be involved. ${ }^{8}$ This is consistent with another study by Deshmukh et al., who showed that femur (43\%) was the most frequently involved site among 7 cases of bone non-Hodgkin lymphoma. ${ }^{9}$ We had the similar finding that thecase presented as bone pain had the involvement of lymphoma over her femur. 
In our case only femur bone was involved presenting as bone pain and no other soft tissue or lymph node involvement in clinico-radiological workup was found.

\section{CONCLUSION}

Lymphomas can develop in any location where lymphomatous tissue exists, but extranodal presentation in the lower limb and pelvis are uncommon. The majority of these are Large B-cell non-Hodgkin lymphomas. The treatment depends on histological type and stage. Radiotherapy and/or chemotherapy is the preferred modality of management.

\section{REFERENCES}

1. El-Zimaty HM, wotherspoonA, de Jong D. On behalf of Houston MALT Lymphoma workshop. Interobserver variation in histological assessment of MALT: Towards a consensces. Blood cells Mol Dis 2005 ;34:6-140 Crossref

2. Parker F, Jackson H. Primary reticulum cell sarcoma of bone. Surg Gynecol Obstet 1939;68:45-53.

3. Huan-Tee Chan, Chi-Chou Tseng, Po-Yih Chen et al. Intraosseous Lymphoma Presenting as a Fracture of the Femoral Neck. International Journal of Case Reports in Medicine,2013 . Article ID 275363. DOI: 10.5171/2013. 275363
4. Siddiqui YS, Khan AQ, Sherwani MKA. Pathological Fractures in primary Non-Hodgkin's Lymphoma of the Bone: A Case Series with Review of the literature. J Clin Diagn Res 2013; 7: 513-7. Crossref

5. Suleman FE.; Primary bone lymphoma: Imaging findings of a rare primary bone tumour. Sa orthopaedic journal Spring 2011;10:68-70.

6. K. M. Ramadan, T. Shenkier, L. H. Sehn, R. D. Gascoyne \& J. M. Connors; A clinicopathological retrospective study of 131 patients with primary bone lymphoma: A population-based study of successively treated cohorts from the British Columbia Cancer Agency. Ann Oncol 2007;18:129-35. Crossref

7. Qureshi A, Ali A, Riaz N, Pervez S. Primary Non-Hodgkin's Lymphoma of Bone: Experience of a Decade. Indian J Pathol Microbiol 2010;53: 267-70. Crossref

8. El-Essawy MT, Vanhoenacker FM, Van Dijck H, Ferrante M. Primary Lymphoma of Iliac Bone. JBR-BTR 2012;95:375. Crossref

9. Deshmukh C, Bakshi A, Parikh P, Nair R, Pai V, Gupta S, et al. Primary non-Hodgkin's lymphoma of the bone: a single institution experience. Med Oncol. 2004; 21:263-8 Crossref 